\title{
Investigating Factors that Influence Math Homework Expectancy: A Multilevel Approach
}

\author{
Jianzhong $\mathrm{Xu}^{1,2}{ }^{1}$, Chuang Wang ${ }^{2, *}$ and Jianxia $\mathrm{Du}^{2}$ \\ 1 Department of Counseling, Educational Psychology, and Foundations, Mississippi State University, \\ Starkville, MS 39759, USA; jx18@colled.msstate.edu or jianzhongxu18@gmail.com \\ 2 Faculty of Education, University of Macau, Macau 999078, China; jianxiadu@umac.mo \\ * Correspondence: wangc@um.edu.mo
}

Received: 13 July 2020; Accepted: 12 August 2020; Published: 14 August 2020

\begin{abstract}
The present study investigated several clusters of variables influencing students' math homework expectancy based on 3018 students in grade 8 in China. Results revealed that math homework expectancy was positively associated with homework quality at both the individual and class level. At the individual level, math homework expectancy was positively associated with prior math knowledge, parent education, homework interest, homework favorability, parent autonomy support, mastery-approach, and homework value. Meanwhile, math homework expectancy was negatively associated with homework time, parent help quantity, and performance-approach. Finally, compared with females, males tended to have higher math homework expectancy.
\end{abstract}

Keywords: achievement motivation theories; expectancy beliefs; homework expectancy; math; middle school; multilevel analysis

\section{Introduction}

According to the expectancy-value theory of academic motivation [1,2], expectancy-relating to the question "Can I do this task?"-is one of the most important motivation constructs that influence achievement-related outcomes such as academic achievement. Given that homework is a widespread achievement-related activity across countries with everyday importance for many students, parents, and teachers [3-5], homework expectancy (i.e., student's belief in being able to successfully complete homework assignments) is highly important for homework research and practice [6,7].

One comprehensive homework model [6] has conceptualized homework expectancy as influenced by four clusters of variables (demographic, homework, teacher, and parent variables). Yet, several important variables that may influence homework expectancy are not explicitly included in Trautwein et al.'s model, such as homework interest, autonomy support, and goal orientations (i.e., types of goals or mindsets students have when doing homework assignments.

The current investigation attempts to bridge these gaps by examining models of math homework expectancy for middle school students. A study such as this is particularly important given the centrality of math competencies in science, technology, engineering, and math (STEM) subjects and STEM-related careers [8], and given the more prominent role of homework expectancy in math than in other subjects (e.g., English) [6]. Furthermore, there is a critical need to focus on middle school students, as math expectancy steadily declines across early adolescent years [9-11].

\subsection{Theoretical Framework}

Expectancy-value theory [1,2] provides a comprehensive framework for understanding human motivation in achievement-related tasks. According to expectancy-value theory, expectancy is a central construct influencing achievement-related outcomes. It refers to the degree to which learners think 
that they can successfully accomplish an achievement-related task. Over time, studies have found that expectancy and value have a positive influence on task engagement, persistence, and academic achievement $[12,13]$. Additionally, expectancy is a stronger predictor of actual performance than value $[2,14]$. Furthermore, expectancy is more strongly associated with achievement as students get older $[10,15]$.

Building on expectancy-value theory [1,11], Trautwein et al. [6] developed a model of factors that influence the effectiveness of homework, with homework motivation (expectancy and value) as the backbone of their homework model. The model adopts a domain-specific and multilevel approach to homework motivation, as human motivation is domain-specific, and as certain teacher and homework characteristics (e.g., homework quality) may influence students' motivation at the student and class levels. Specifically, this model postulates that student characteristics (e.g., gender and prior knowledge), homework characteristics (e.g., homework time, frequency, and quality), teacher characteristics (e.g., feedback), and the role of parents (e.g., quantity and quality of help) influence students' homework motivation (expectancy and value). Additionally, it postulates that homework motivation, in turn, influences homework behavior (e.g., effort and completion) and student achievement. Due to the link to the present investigation objectives, we focus on clusters of variables that may affect homework expectancy.

Congruent with expectancy-value theory [1,11], Trautwein et al.'s model [6] further postulates that homework expectancy and value (i.e., the extent to which students think homework is worthwhile) are related. Hence, according to this model, homework expectancy could be influenced by homework value.

Although Trautwein et al. [6] do not explicitly tap into achievement goal orientations in their homework model, expectancy-value theory posits that the expectation of success (i.e., expectancy) is influenced by individuals' goals (e.g., goals for learning activities) [11]. As there is growing evidence regarding the positive influence of mastery-approach on self-efficacy [16,17], as few studies have integrated constructs from expectancy-value theory and achievement goal theory [18], it would be informative to study the predictive effect of homework goal orientations (mastery- and performance-approaches) on homework expectancy.

To sum up, this body of literature suggests that homework expectancy is affected by several clusters of variables. They include demographic, homework, teacher, parent, and other motivation variables (homework goal orientations and homework value).

\subsection{Related Literature}

Informed by Trautwein et al.'s homework model [6], a number of studies have tapped into several variables that may influence homework expectancy, including gender [19-21], prior achievements or cognitive abilities [6,19], parent education [19], homework quality [19,20], parental homework help [20,21], and parental homework control [6,20].

Results revealed that, compared with females, males tended to have higher math homework expectancy [6,19]. Math homework expectancy was positively associated with homework quality at the student and class levels $[19,20]$. Additionally, at the student level, math homework expectancy was positively related to prior achievements and cognitive abilities, but unrelated to socioeconomic status (SES) and parent education [19]. Furthermore, math homework expectancy was negatively related to parental homework control, but unrelated to teacher homework control and parental homework help [20].

Other studies have linked homework expectancy to academic achievement $[7,19,22]$. In line with related studies based on expectancy-value theory [2,14], results from these homework studies revealed that homework expectancy (in comparison with homework value) was a stronger predictor of math achievement. 


\subsection{The Present Study}

Taken together, a number of studies suggest several variables that may influence math homework expectancy (e.g., gender, prior knowledge, parent education, homework quality, and parental homework control) $[6,19,20]$. However, extant studies did not include other important variables in Trautwein et al.'s homework model [6] that may influence homework expectancy.

First, other than homework quality, extant studies did not include or control two homework variables in Trautwein et al.'s model [6] that may influence homework expectancy (i.e., homework time and homework frequency). Whereas Trautwein et al.'s model [6] does not include homework interest and favorability (one's favorite attitude toward homework as compared with alternative activities), it would be important to test their predictive effects on homework expectancy, because interest has a positive influence on competence beliefs $[23,24]$ and because homework favorability and interest as two empirically distinguishable constructs have a large positive correlation with each other $[25,26]$.

Second, extant studies did not incorporate other motivational variables such as mastery-approach, performance-approach, and homework value. Given our previous discussion concerning the predictive effects of these variables on homework expectancy $[6,16,18]$, it would be informative to incorporate these variables in the current investigation.

Third, in line with Trautwein et al.'s model [6], recent literature points to the importance of incorporating the quality of teacher and parent variables in the homework process. These variables include the quality of teacher involvement (e.g., autonomy support and teacher feedback quality) $[27,28]$ and the quality of parental help (e.g., autonomy support and content support) [29,30]. Although no systematic attempt has been made to link these "quality" variables (e.g., teacher autonomy support and parent autonomy support) to homework expectancy, there is a need to incorporate these variables in the current study, as concurrent validity evidence in validation studies [28,30] revealed that parent autonomy support, parent content support, teacher autonomy support, and teacher feedback quality were positively correlated with homework expectancy.

To bridge these important gaps in the extant literature, the current study adopted a multilevel research model as a conceptual framework to investigate math homework expectancy at two levels: the individual level and the class level. Specifically, we addressed the following two sets of research questions.

The first set of research questions targeted five clusters of variables at the individual level. Is math homework expectancy associated with demographic variables (gender, prior knowledge, and parent education), homework variables (time, frequency, quality, interest, favorability), teacher variables (feedback quantity, feedback quality, and autonomy support), and parent variables (frequency of help, content support, and autonomy support), and other motivation variables (mastery-approach, performance-approach, and value)?

The second set of research questions concerned two clusters of variables (i.e., homework and teacher variables) at the class level. Is math homework expectancy related to homework variables (time, frequency, quality, interest, and favorability) and teacher variables (feedback quantity, feedback quality, and autonomy support)?

\section{Materials and Methods}

\subsection{Participants and Procedure}

Three thousand and eighteen 8 th graders (45.6\% female; $100 \%$ Han nationality) participated in our study. They came from 96 classes from a number of regions in China (e.g., southeastern, central, and southeastern). Overall, the educational level was 11.4 years and 10.6 years for fathers and mothers, respectively, and they were significantly correlated $(r=76, p<001)$.

With respect to math homework practices, $76.9 \%$ students did math homework four or more days weekly. On average, they spent $34.1 \mathrm{~min}(S D=24.7)$ on math homework a day. These practices are similar to recent homework studies in China [30]. 
We obtained permission from parents and teachers for students to participate in our current investigation. A number of research assistants administered the instrument (see below) in math classes during regular school hours at the end of October, after students had about eight weeks of experience with math homework assignments at this grade level. Teachers were asked to leave their classrooms during the instrument administration. Overall, the response rate was $88.7 \%$.

\subsection{Instruments}

Participants were asked how often they received math homework from their teachers, from none (1) to five or more days a week (5). They were also asked about the time spent doing math homework daily, including none (1), 1-20 min (2), 21-40 $\mathrm{min}$ (3), 41-60 min (4), 61-80 $\mathrm{min}$ (5), 81-100 $\mathrm{min}$ (6), 101 to $130 \mathrm{~min}$ (7), and more than $120 \mathrm{~min}$ (8). Following a previous related study [31], a variable of time on math homework was developed by converting each response to its midpoint (e.g., $1=0$ min; $2=10.5 \mathrm{~min}$; and $3=30.5 \mathrm{~min}$ ).

Participants indicated the frequency of parent homework help, from never (1) to routinely (5). They were also asked about education level for mother and father. A variable concerning parent education was generated by averaging each parent's education. Additionally, prior math knowledge was based on students' math grade at the end of grade 7, including $F(1), D(2), C(3), B(4)$, and $A$ (5).

Furthermore, we used multiple scales in our current study. Table 1 contains sample items from these scales and their reliability coefficients.

Table 1. Reliability Estimates of Multi-Item Scales.

\begin{tabular}{|c|c|c|c|c|}
\hline Scales & $\mathbf{N}$ & Sample Items & $\alpha$ & $\omega$ \\
\hline HW quality ${ }^{a}$ & 4 & $\begin{array}{l}\text { Our math HW assignments really help us to understand our } \\
\text { math lessons. } \\
\text { Our math HW assignments are always well integrated into } \\
\text { the lessons. }\end{array}$ & 0.87 & 0.87 \\
\hline HW interest ${ }^{b}$ & 4 & $\begin{array}{l}\text { I look forward to math HW } \\
\text { I enjoy math HW. }\end{array}$ & 0.91 & 0.91 \\
\hline HW favorability & 3 & $\begin{array}{l}\text { My motivation to do math } \mathrm{HW} \text { is ___ }{ }^{\mathrm{c}} \text { other after-school } \\
\text { activities. } \\
\text { My attention while doing math } \mathrm{HW} \text { is __ }{ }^{\mathrm{c}} \text { other } \\
\text { after-school activities. }\end{array}$ & 0.83 & 0.83 \\
\hline $\begin{array}{l}\text { Teacher feedback } \\
\text { quantity } d\end{array}$ & 3 & $\begin{array}{l}\text { How much of your math HW is checked by the math } \\
\text { teacher? } \\
\text { How much of your math HW is graded by the math teacher? }\end{array}$ & 0.70 & 0.71 \\
\hline $\begin{array}{l}\text { Teacher feedback quality } \\
\text { a }\end{array}$ & 4 & $\begin{array}{l}\text { I value the feedback I receive from my math teacher. } \\
\text { My math teacher consistently provides me with useful } \\
\text { information about my HW performance. }\end{array}$ & 0.87 & 0.88 \\
\hline $\begin{array}{l}\text { Teacher autonomy } \\
\text { support }^{\text {a }}\end{array}$ & 4 & $\begin{array}{l}\text { My math teacher encourages me to ask questions about HW } \\
\text { assignments. } \\
\text { My math teacher listens to my ideas about HW assignments. }\end{array}$ & 0.83 & 0.83 \\
\hline Parent content support ${ }^{\mathrm{a}}$ & 4 & $\begin{array}{l}\text { My parents often ask how they can help me with my } \\
\text { math HW. } \\
\text { My parents always help me if I get stuck with my math HW. }\end{array}$ & 0.87 & 0.87 \\
\hline $\begin{array}{l}\text { Parent autonomy } \\
\text { support }^{\text {a }}\end{array}$ & 4 & $\begin{array}{l}\text { My parents encourage me to ask questions about HW } \\
\text { assignments. } \\
\text { My parents listen to my ideas about HW assignments. }\end{array}$ & 0.90 & 0.90 \\
\hline Mastery-approach ${ }^{\mathrm{e}}$ & 4 & $\begin{array}{l}\text { I want to learn as much as possible in math homework. } \\
\text { I prefer math homework that really challenges me so I can } \\
\text { learn new things. }\end{array}$ & 0.86 & 0.86 \\
\hline
\end{tabular}


Table 1. Cont.

\begin{tabular}{|c|c|c|c|c|}
\hline Scales & $\mathbf{N}$ & Sample Items & $\alpha$ & $\omega$ \\
\hline Performance-approach ${ }^{\mathrm{e}}$ & 3 & $\begin{array}{l}\text { My goal in doing math HW is to get a better grade than } \\
\text { most of the other students. } \\
\text { I want to do well in math HW to show my ability to my } \\
\text { family, friends, teachers, or others. }\end{array}$ & 0.76 & 0.76 \\
\hline HW value ${ }^{a}$ & 4 & $\begin{array}{l}\text { I don't learn much from our math } \mathrm{HW}^{\mathrm{f}} \text {. } \\
\text { There is no point in my doing math } \mathrm{HW}^{\mathrm{f}} \text {. }\end{array}$ & 0.84 & 0.85 \\
\hline HW expectancy ${ }^{a}$ & 4 & $\begin{array}{l}\text { Whether or not I do my math HW, I don't understand a } \\
\text { thing in the lesson anyway }{ }^{\mathrm{f}} \text {. } \\
\text { I sometimes really dread math } \mathrm{HW}^{\mathrm{f}} \text {. }\end{array}$ & 0.80 & 0.80 \\
\hline
\end{tabular}

Note: $\mathrm{N}=$ number of items. $\mathrm{HW}=$ homework. ${ }^{\mathrm{a}}$ Rating: Strongly disagree $=1$, Disagree $=2$, Agree $=3$, Strongly agree $=4 .{ }^{\mathrm{b}}$ Rating: Strongly disagree $=1$, Disagree $=2$, Neither disagree nor agree $=3$, Agree $=4$, Strongly agree $=5$.

c Rating: Much lower than =1, Lower than =2, About the same as $=3$, Higher than $=4$, Much higher than $=5$.

d Rating: None $=1$, Some $=2$, About half $=3$, Most $=4$, All $=5$. ${ }^{\text {e }}$ Rating: Not at all true of me $=1$ to Very true of $\mathrm{me}=7 .{ }^{\mathrm{f}}$ Item was reverse scored.

\subsubsection{Homework Quality}

Homework quality contained four items to measure students' perceptions of the quality of math homework assigned by teachers [28]. It focused on the degree to which homework was integrated into math classes and helped participants to better understand math concepts taught in class.

\subsubsection{Homework Interest}

Homework interest included four items to measure students' interest in math assignments, drawn from the extant literature on interest and intrinsic motivation $[32,33]$ and previous homework studies [25,31]. It measured the extent to which students enjoyed math homework.

\subsubsection{Homework Favorability}

Homework favorability included three items to assess students' favorability of math homework, based on the extant literature [25,34-36]. It measured their affective attitude toward math homework, in comparison with their experience with other after-school activities (e.g., regarding motivation, moods, and attention).

\subsubsection{Teacher Feedback Quantity}

Drawn from related homework literature [6,37], this scale incorporated three items to assess how frequently math homework was checked, collected, and graded by teachers.

\subsubsection{Teacher Feedback Quality}

Teacher feedback quality contained four items to measure the quality of the math homework feedback offered by teachers [28]. It examined the degree to which teachers offered helpful and useful feedback concerning math homework assignments.

\subsubsection{Teacher Autonomy Support}

Teacher autonomy support contained four items to assess autonomy support during the homework process [28]. It focused on students' perspectives of teachers' role as autonomy supportive concerning math homework (e.g., listening to their ideas about how to approach math homework and promoting their initiatives). 


\subsubsection{Parent Content Support}

Parent content support contained four items to measure content support by parents [30]. It measured the degree to which parents provided direct assistance on math homework when asked by students (e.g., when students experienced difficulty in completing their assignments).

\subsubsection{Parent Autonomy Support}

Parent autonomy support included four items to measure autonomy support by parents [30]. It focused on students' perspectives of parents' autonomy support regarding math homework (e.g., listening to their ideas about how to do math homework and encouraging their initiatives).

\subsubsection{Homework Goal Orientation}

We assessed mastery- and performance-approaches using the Homework Goal Orientation Scale [38]. Four items measured students' adoption of mastery-approach regarding math homework (e.g., to better understand math concepts). Three items measured students' adoption of performance-approach concerning math homework (e.g., to do math homework better than others).

\subsubsection{Homework Value}

We assessed homework value using the Homework Expectancy Value Scale $[7,33]$. Four items assessed students' value belief concerning math homework (e.g., the utility and importance of math and math homework).

\subsubsection{Homework Expectancy}

We assessed homework expectancy using the Homework Expectancy Value Scale [7,22]. Four items measured students' expectancy belief concerning math homework (e.g., self-confidence to follow through and complete math assignments).

Regarding the predictive validity evidence of homework expectancy, in a previous validation study with 918 students in grades 7-9, Yang and $\mathrm{Xu}$ [22] found that math homework expectancy was positively related to math achievement $(r=0.24, p<0.01)$. Similarly, in our study, math homework expectancy was positively related to math achievement at the end of the school year $(r=0.31, p<0.01)$.

\subsection{Data Analysis}

As students were nested in classes, we used a multilevel approach to address relevant issues resulting from this nested data structure (e.g., non-independent observations) [39]. To aid the interpretation of our findings, we standardized all continuous variables. Hence, the regression weights for these variables were comparable with the standardized weights using multiple regression procedures [25]

Model 1 contained seventeen student-level variables. It included demographic variables (gender, prior math knowledge, parent education), homework variables (time, frequency, quality, interest, and favorability), teacher variables (feedback quantity, feedback quality, and autonomy support), parent variables (frequency of help, content support, and autonomy support), homework goal orientation (mastery- and performance-approaches), and homework value. Model 2 included eight class-level variables, including homework (time, frequency, quality, interest, and favorability) and teacher variables (feedback quantity, feedback quality, and autonomy support).

All models in our study were random-intercept models [39], as we do not have any hypotheses regarding the predictive power of the level-1 predictors across the classes. The models are presented below. For math homework expectancy $Y_{i j}$, level 1 takes the following form:

$$
Y_{i j}=\beta_{0 j}+\beta_{1 j}(\text { Gender })_{i j}+\beta_{2 j}(\text { Parent education })_{i j}+\ldots+\beta_{17 j}(\text { Homework value })_{i j}+r_{i j}
$$


where $Y_{i j}$ is the math homework expectancy measure for student $i$ nested in class $j$. The variability among the classes in the model intercept $\left(\beta_{0 j}\right)$ may be affected by class-level predictors, as indicated in level 2 below:

$$
\begin{gathered}
\beta_{0 j}=\gamma_{00}+\gamma_{01} \text { (Homework time) }+\gamma_{02} \text { (Homework frequency) }+\ldots+\gamma_{08} \text { (Teacher } \\
\text { autonomy support })+\mu_{0 j}
\end{gathered}
$$

To separate compositional and individual effects, all eight class-level variables were centered at the group mean [39]. Full maximum likelihood was applied in our investigation. Missing values for the current investigation varied from $0 \%$ to $5.14 \%(M=1.49 \%)$, and they were imputed using the expectation-maximization approach.

\section{Results}

\subsection{Preliminary Analyses}

Although Likert-type items are technically ordinal, Likert scales (consisting of means or sums across multiple items) can be viewed as an interval $[40,41]$. Although parametric statistical methods are based on an assumption of normally distributed interval data, Norman [41] has found that parametric statistics are robust with respect to violations of these assumptions. In the case of hierarchical linear models, provided with reasonably normal distributions, the results should not be affected [39,42]. In our investigation, the skewness and kurtosis values for all Likert-type scales can be viewed as quite satisfactory; only one scale with kurtosis value was slightly larger than the absolute value 1 (i.e., 1.05 for homework quality).

As we incorporated eight class-level variables by aggregating students' ratings of their learning environments within each class (regarding homework and teacher characteristics), it would be important to examine whether the aggregation of student ratings to the class level could be reliably assessed [42]. Results indicated that the aggregate reliabilities for five class-level homework variables were 0.71 (time), 0.92 (frequency), 0.81 (quality), 0.74 (interest), and 0.74 (favorability). The aggregated reliabilities for three class-level teacher variables were 0.84 (feedback quantity), 0.64 (feedback quality), and 0.75 (autonomy support). As a value of 60 is viewed as a lower bound for acceptable reliability of aggregated ratings [20], we were able to justify the aggregation of student ratings to the class level.

Table 2 displays the means, standard deviations, and Pearson correlations of all variables. Math homework expectancy was significantly associated with all predictor variables, except homework time at the individual level. 
Table 2. Descriptive Statistics and Correlations.

\begin{tabular}{|c|c|c|c|c|c|c|c|c|c|c|c|c|c|c|c|c|c|c|c|c|c|c|c|c|c|c|c|}
\hline Variables & $\mathrm{M}$ & SD & 1 & 2 & 3 & 4 & 5 & 6 & 7 & 8 & 9 & 10 & 11 & 12 & 13 & 14 & 15 & 16 & 17 & 18 & 19 & 20 & 21 & 22 & 23 & 24 & 25 \\
\hline 1 Gender (male: 1 ) & 54 & 0.50 & - & & & & & & & & & & & & & & & & & & & & & & & & \\
\hline $\begin{array}{l}2 \text { Prior math } \\
\text { knowledge }\end{array}$ & 3.01 & 1.34 & -0.01 & - & & & & & & & & & & & & & & & & & & & & & & & \\
\hline 3 Parent education & 10.99 & 3.14 & -0.01 & $0.34^{+}$ & - & & & & & & & & & & & & & & & & & & & & & & \\
\hline $4 \mathrm{HW}$ time & 34.12 & 24.74 & 0.00 & $0.10^{+}$ & 0.03 & - & & & & & & & & & & & & & & & & & & & & & \\
\hline $5 \mathrm{HW}$ frequency & 5.20 & 1.33 & -0.02 & $0.22^{\dagger}$ & $0.14^{+}$ & $0.12^{+}$ & - & & & & & & & & & & & & & & & & & & & & \\
\hline 6 HW quality & 3.15 & 0.61 & $-0.09^{+}$ & $0.28^{+}$ & $0.16^{+}$ & $0.04 *$ & $0.24^{+}$ & - & & & & & & & & & & & & & & & & & & & \\
\hline $7 \mathrm{HW}$ interest & 3.22 & 0.89 & -0.01 & $0.34^{+}$ & $0.13^{+}$ & $0.09^{+}$ & $0.15^{+}$ & $0.43^{+}$ & - & & & & & & & & & & & & & & & & & & \\
\hline $8 \mathrm{HW}$ favorability & 2.83 & 0.90 & -0.02 & $0.32^{+}$ & $0.12^{+}$ & $0.10^{+}$ & $0.13^{+}$ & $0.32^{+}$ & $0.61^{+}$ & - & & & & & & & & & & & & & & & & & \\
\hline $\begin{array}{l}9 \text { Teacher feedback } \\
\text { quantity }\end{array}$ & 3.32 & 1.04 & 0.03 & $0.13^{+}$ & 0.02 & 0.01 & $0.11^{+}$ & $0.22^{+}$ & $0.19^{+}$ & $0.17^{+}$ & - & & & & & & & & & & & & & & & & \\
\hline $\begin{array}{l}10 \text { Teacher feedback } \\
\text { quality }\end{array}$ & 2.75 & 0.69 & -0.02 & $0.18^{+}$ & $0.10^{+}$ & $0.05^{+}$ & $0.12^{+}$ & $0.38^{+}$ & $0.37^{+}$ & $0.29^{+}$ & $0.16^{+}$ & - & & & & & & & & & & & & & & & \\
\hline $\begin{array}{l}11 \text { Teacher autonomy } \\
\text { support }\end{array}$ & 2.67 & 0.66 & -0.01 & $0.17^{+}$ & $0.13^{+}$ & $0.06^{+}$ & $0.11^{+}$ & $0.34^{+}$ & $0.33^{+}$ & $0.28^{+}$ & $0.13^{+}$ & $0.62^{+}$ & - & & & & & & & & & & & & & & \\
\hline 12 Parent help quantity & 2.71 & 1.06 & $0.06^{+}$ & $0.08^{+}$ & $0.23^{+}$ & $0.07^{+}$ & $0.06^{+}$ & $0.10^{+}$ & $0.21^{+}$ & $0.22^{+}$ & $0.16^{+}$ & $0.21^{+}$ & $0.20^{+}$ & - & & & & & & & & & & & & & \\
\hline $\begin{array}{l}13 \text { Parent content } \\
\text { support }\end{array}$ & 2.53 & 0.70 & 0.02 & $0.09^{\dagger}$ & $0.25^{+}$ & $0.05^{+}$ & $0.08^{+}$ & $0.20^{+}$ & $0.29^{+}$ & $0.25^{+}$ & $0.17^{\dagger}$ & $0.26^{+}$ & $0.28^{+}$ & $0.57^{+}$ & - & & & & & & & & & & & & \\
\hline $\begin{array}{l}14 \text { Parent autonomy } \\
\text { support }\end{array}$ & 2.67 & 0.76 & $-0.06^{+}$ & $0.18^{+}$ & $0.20^{+}$ & $0.05^{+}$ & $0.10^{+}$ & $0.23^{+}$ & $0.30^{+}$ & $0.25^{+}$ & $0.15^{+}$ & $0.39^{+}$ & $0.46^{+}$ & $0.38^{+}$ & $0.50^{+}$ & - & & & & & & & & & & & \\
\hline 15 Mastery-approach & 5.02 & 1.58 & $-0.08^{+}$ & $0.42^{+}$ & $0.21^{+}$ & $0.07^{+}$ & $0.21^{+}$ & $0.40^{+}$ & $0.47^{+}$ & $0.39^{+}$ & $0.13^{+}$ & $0.39^{+}$ & $0.33^{+}$ & $0.14^{+}$ & $0.20^{+}$ & $0.31^{+}$ & - & & & & & & & & & & \\
\hline $\begin{array}{l}16 \\
\text { Performance-approach }\end{array}$ & 4.32 & 1.60 & $0.04 *$ & $0.24^{+}$ & $0.16^{+}$ & 0.03 & $0.14^{+}$ & $0.19^{+}$ & $0.27^{+}$ & $0.24^{+}$ & $0.11^{+}$ & $0.27^{+}$ & $0.25^{+}$ & $0.16^{+}$ & $0.18^{+}$ & $0.23^{+}$ & $0.48^{+}$ & - & & & & & & & & & \\
\hline $17 \mathrm{HW}$ value & 3.22 & 0.64 & $-0.09^{+}$ & $0.28^{+}$ & $0.07^{+}$ & 0.02 & $0.18^{\dagger}$ & $0.40^{\circ}$ & $0.39^{+}$ & $0.30^{+}$ & $0.10^{+}$ & $0.27^{+}$ & $0.21^{+}$ & $0.06^{+}$ & $0.10^{+}$ & $0.19^{+}$ & $0.43^{+}$ & $0.16^{+}$ & - & & & & & & & & \\
\hline $18 \mathrm{HW}$ time-C & 34.22 & 8.34 & $-0.07^{+}$ & $0.21^{\dagger}$ & $0.05^{+}$ & $0.35^{\dagger}$ & & $0.06^{+}$ & $0.08^{+}$ & $0.09^{+}$ & 0.03 & & $0.07^{+}$ & 0.01 & -0.04 & $0.06^{+}$ & $0.13^{\dagger}$ & 0.02 & $0.12^{+}$ & - & & & & & & & \\
\hline $19 \mathrm{HW}$ frequency-C & 5.21 & 0.77 & 0.02 & $0.27^{+}$ & $0.21^{+}$ & $0.10^{+}$ & $0.57^{\dagger}$ & $0.22^{+}$ & $0.13^{+}$ & $0.11^{+}$ & $0.08^{+}$ & $0.13^{+}$ & $0.13^{+}$ & 0.03 & 0.00 & $0.08^{+}$ & $0.19^{\dagger}$ & $0.10^{\dagger}$ & $0.18^{+}$ & $0.30^{+}$ & - & & & & & & \\
\hline $20 \mathrm{HW}$ quality-C & 3.20 & 0.25 & -0.02 & $0.38^{+}$ & $0.34^{+}$ & $0.05^{+}$ & $0.30^{\dagger}$ & $0.42^{+}$ & $0.24^{+}$ & $0.16^{+}$ & $0.09^{+}$ & $0.21^{+}$ & $0.22^{+}$ & 0.02 & 0.03 & $0.15^{+}$ & $0.32^{\dagger}$ & $0.13^{+}$ & $0.30^{+}$ & $0.15^{+}$ & $0.53^{+}$ & - & & & & & \\
\hline $21 \mathrm{HW}$ interest-C & 3.28 & 0.33 & -0.03 & $0.39^{\dagger}$ & $0.24^{+}$ & $0.08^{+}$ & $0.21^{\dagger}$ & $0.29^{+}$ & $0.35^{+}$ & $0.28^{+}$ & $0.14^{+}$ & $0.23^{+}$ & $0.23^{+}$ & $0.13^{+}$ & $0.12^{+}$ & $0.17^{+}$ & $0.31^{\dagger}$ & $0.16^{+}$ & $0.26^{+}$ & $0.24^{\dagger}$ & $0.37^{+}$ & $0.69^{+}$ & - & & & & \\
\hline $22 \mathrm{HW}$ favorability-C & 2.87 & 0.36 & -0.02 & $0.35^{+}$ & $0.20^{+}$ & $0.09^{+}$ & $0.18^{+}$ & $0.20^{+}$ & $0.28^{+}$ & $0.34^{+}$ & $0.20^{+}$ & $0.19^{+}$ & $0.20^{+}$ & $0.16^{+}$ & $0.14^{+}$ & $0.17^{+}$ & $0.26^{+}$ & $0.18^{+}$ & $0.22^{+}$ & $0.27^{+}$ & $0.32^{+}$ & $0.47^{+}$ & $0.81^{\dagger}$ & - & & & \\
\hline $\begin{array}{l}23 \text { Teacher feedback } \\
\text { quantity-C }\end{array}$ & 3.33 & 0.47 & 0.01 & $0.17^{+}$ & $0.06^{+}$ & 0.02 & $0.10^{+}$ & $0.08^{+}$ & $0.11^{+}$ & $0.15^{+}$ & $0.45^{+}$ & $0.07^{+}$ & $0.06^{+}$ & $0.08^{+}$ & $0.06^{+}$ & $0.08^{+}$ & $0.06^{+}$ & $0.07^{\dagger}$ & $0.06^{+}$ & $0.06^{+}$ & $0.18^{+}$ & $0.19^{+}$ & $0.31^{+}$ & $0.43^{+}$ & - & & \\
\hline $\begin{array}{l}24 \text { Teacher feedback } \\
\text { quality-C }\end{array}$ & 2.77 & 0.22 & -0.01 & $0.27^{+}$ & $0.20^{+}$ & $0.04 *$ & $0.25^{+}$ & $0.29^{+}$ & $0.26^{+}$ & $0.22^{+}$ & $0.11^{+}$ & $0.31^{+}$ & $0.27^{+}$ & $0.07^{+}$ & $0.08^{+}$ & $0.15^{+}$ & $0.27^{+}$ & $0.16^{+}$ & $0.24^{+}$ & $0.13^{+}$ & $0.43^{+}$ & $0.68^{+}$ & $0.76^{+}$ & $0.63^{+}$ & $0.24^{+}$ & - & \\
\hline $\begin{array}{l}25 \text { Teacher autonomy } \\
\text { support-C }\end{array}$ & 2.70 & 0.26 & -0.02 & $0.28^{+}$ & $0.28^{+}$ & $0.07^{+}$ & $0.21^{+}$ & $0.27^{+}$ & $0.22^{+}$ & $0.20^{+}$ & $0.08^{+}$ & $0.24^{+}$ & $0.35^{+}$ & $0.10^{+}$ & $0.11^{+}$ & $0.21^{+}$ & $0.29^{+}$ & $0.18^{+}$ & $0.23^{+}$ & $0.21^{+}$ & $0.37^{+}$ & $0.63^{+}$ & $0.64^{+}$ & $0.57^{+}$ & $0.18^{+}$ & $0.78^{+}$ & - \\
\hline $26 \mathrm{HW}$ expectancy & 3.03 & 0.68 & $0.07^{+}$ & $0.43^{+}$ & $0.18^{+}$ & -0.01 & $0.14^{+}$ & $0.32^{+}$ & $0.40^{+}$ & $0.33^{+}$ & $0.09^{+}$ & $0.18^{+}$ & $0.16^{+}$ & 0.04 * & $0.12^{+}$ & $0.18^{+}$ & $0.37^{+}$ & $0.15^{+}$ & $0.41^{\dagger}$ & $0.07^{+}$ & $0.17^{+}$ & $0.29^{+}$ & $0.24^{+}$ & $0.18^{+}$ & $0.07^{+}$ & $0.20^{+}$ & $0.19^{+}$ \\
\hline
\end{tabular}

Note: $\mathrm{HW}=$ homework. $\mathrm{C}=$ class. $N=3018 .{ }^{*} p<0.05 .{ }^{\dagger} p<0.01$. 


\subsection{Multilevel Analyses}

The null model was carried out to estimate the variance in math homework expectancy at the individual and class levels. Our data showed that $13.2 \%$ of the variance in math homework expectancy was located at the class level. For the model, the deviance statistics was 8330.367 ( 3 parameters estimated).

As shown in Table 3, Model 1 introduced 17 student-level variables. The deviance statistics for Model 1 was 7293.133 (20 parameters estimated). The likelihood ratio test comparing Model 1 and the null model was highly significant $\left[\chi^{2}(17)=1037.234, p<0.001\right]$, indicating a significant improvement of Model 1 over the null model. Model 1 explained 27.1\% of the variance in math homework expectancy at the individual level and $79.9 \%$ of the variance at the class level.

Table 3. Multilevel Modeling Results for Math Homework Expectancy.

\begin{tabular}{|c|c|c|c|c|c|}
\hline \multirow{2}{*}{ Model Predictor } & Null Model & \multicolumn{2}{|c|}{ Model 1} & \multicolumn{2}{|c|}{ Model 2} \\
\hline & $S E$ & $b$ & $S E$ & $b$ & $S E$ \\
\hline \multicolumn{6}{|l|}{ Individual level } \\
\hline Gender (female: 0 , male: 1 ) & & $0.23^{* * *}$ & 0.03 & $0.22 * * *$ & 0.03 \\
\hline Prior math knowledge & & $0.26^{* * *}$ & 0.02 & $0.25^{* * *}$ & 0.02 \\
\hline Parent education & & 0.03 & 0.02 & 0.02 & 0.02 \\
\hline Homework time & & $-0.06^{* *}$ & 0.02 & $-0.06^{* *}$ & 0.02 \\
\hline Homework frequency & & -0.01 & 0.02 & 0.00 & 0.02 \\
\hline Homework quality & & $0.05 *$ & 0.02 & $0.05 *$ & 0.02 \\
\hline Homework interest & & $0.14^{* * *}$ & 0.02 & $0.14^{* * *}$ & 0.02 \\
\hline Homework favorability & & $0.09 * * *$ & 0.02 & $0.09^{* * *}$ & 0.02 \\
\hline Teacher feedback quantity & & -0.01 & 0.02 & -0.01 & 0.02 \\
\hline Teacher feedback quality & & -0.03 & 0.02 & -0.03 & 0.02 \\
\hline Teacher autonomy support & & -0.04 & 0.02 & -0.04 & 0.02 \\
\hline Parent help quantity & & $-0.07^{* *}$ & 0.02 & $-0.07^{* *}$ & 0.02 \\
\hline Parent content support & & 0.03 & 0.02 & 0.03 & 0.02 \\
\hline Parent autonomy support & & $0.05 *$ & 0.02 & $0.05 *$ & 0.02 \\
\hline Mastery-approach & & $0.10^{* * *}$ & 0.02 & $0.09^{* * *}$ & 0.02 \\
\hline Performance-approach & & $-0.05^{* *}$ & 0.02 & $-0.05^{* *}$ & 0.02 \\
\hline Homework value & & $0.22 * * *$ & 0.02 & $0.22 * * *$ & 0.02 \\
\hline \multicolumn{6}{|l|}{ Class level } \\
\hline Homework time & & & & -0.09 & 0.06 \\
\hline Homework frequency & & & & 0.02 & 0.04 \\
\hline Homework quality & & & & $0.25^{* *}$ & 0.08 \\
\hline Homework interest & & & & 0.16 & 0.13 \\
\hline Homework favorability & & & & -0.20 & 0.14 \\
\hline Teacher feedback quantity & & & & -0.01 & 0.06 \\
\hline Teacher feedback quality & & & & -0.04 & 0.11 \\
\hline Teacher autonomy support & & & & -0.01 & 0.14 \\
\hline$R^{2}$ individual level & & \multicolumn{2}{|c|}{0.271} & \multicolumn{2}{|c|}{0.273} \\
\hline$R^{2}$ class level & & \multicolumn{2}{|c|}{0.799} & \multicolumn{2}{|c|}{0.871} \\
\hline$R^{2}$ total & & \multicolumn{2}{|c|}{0.340} & \multicolumn{2}{|c|}{0.352} \\
\hline Deviance statistics & 8330.367 & \multicolumn{2}{|c|}{7293.133} & \multicolumn{2}{|c|}{7264.043} \\
\hline $\begin{array}{l}\text { Number of estimated } \\
\text { parameters }\end{array}$ & 3 & \multicolumn{2}{|c|}{20} & \multicolumn{2}{|c|}{28} \\
\hline
\end{tabular}

Note. $N=3018$ from 96 classes. $b=$ unstandardized regression coefficient. $S E=$ standard error of b. $R^{2}=$ amount of explained variance. ${ }^{*} p<0.05 .{ }^{* *} p<0.01$. ${ }^{* * *} p<0.001$.

Model 2 incorporated eight class-level variables (see Table 3). The deviance statistics for Model 2 was 7264.043 (28 parameters estimated). The likelihood ratio test comparing Model 2 and Model 1 was highly significant $\left[\chi^{2}(8)=29.090, p<0.001\right]$, showing a significant improvement of Model 2 over Model 1. At the class level, Model 2 explained an extra $7.2 \%$ of the variance in math homework expectancy. 
Overall, Model 2 accounted for $27.3 \%$ of the individual level variance in math homework expectancy, $87.1 \%$ of the class level variance, and a total of $35.2 \%$ of the variance. Regarding demographic variables, math homework expectancy was positively related to prior math knowledge and parent education. Compared with females, males had higher math homework expectancy.

Math homework expectancy was significantly associated with four homework variables at the individual level—negatively with homework time but positively with homework interest, favorability, and quality. Additionally, it was positively related to homework quality at the class level. Concerning parent variables, it is interesting to note that math homework expectancy was unrelated to content support but negatively related to help quantity and positively to autonomy support. Finally, math homework expectancy was positively related to math homework value and mastery-approach, but negatively to performance-approach.

\section{Discussion}

Guided by expectancy and value theory [1,2], Trautwein et al.'s homework model [21], and previous related studies $[19,20]$, the present study investigated multilevel models of homework expectancy that take into consideration five clusters of variables-demographic, homework, teacher, parent, and other motivation variables. In the section below, we organize the discussion according to the different clusters.

\subsection{Demographic Variables}

Our findings concerning demographic variables (gender, prior knowledge, and parent education) are in line with previous studies. The finding that males tended to have higher math expectancy is congruent with previous studies $[19,20]$. Similarly, the finding that math homework expectancy was positively associated with prior math knowledge is congruent with previous studies $[19,20]$. Likewise, the finding that math homework expectancy was unrelated to parent education is largely congruent with a previous study [19]. Given that extant studies were conducted in Germany, our study, based on Chinese students, extends this line of research by implying that these results regarding demographic variables may be applicable across different countries.

\subsection{Homework Variables}

Congruent with prior studies $[19,20]$, our current investigation showed that math homework expectancy was positively associated with homework quality at the individual and class levels. In addition, our study extends the extant studies by linking homework expectancy to four additional homework variables (i.e., time, frequency, interest, and favorability). The finding that homework expectancy was positively associated with homework interest and favorability is not surprising, as it is in line with the extant literature in that interest has a positive influence on competence beliefs [23,24], and in that homework favorability and interest as two distinct constructs are strongly positively correlated $[25,43]$ or positively reciprocally related [44]. Another related explanation is that more self-regulated learning is likely to occur in favorite courses [45,46] or achievement-related activities [26], which in turn serve to enhance student motivation [47].

Meanwhile, how do we explain the fact that math homework expectancy was negatively related to homework time and unrelated to homework frequency? One plausible explanation is that Chinese students are typically assigned math homework on a daily basis. Hence, the influence of homework frequency on math homework expectancy may be less apparent for Chinese school students. Meanwhile, spending more time on math homework assignments may indicate that students have difficulties with these assignments (i.e., low homework expectancy).

\subsection{Teacher Variables}

How do we explain the fact that math homework expectancy was unrelated to teacher variables (feedback quantity, feedback quality, and autonomy support)? Whereas no previous study has explicitly 
linked math homework expectancy to teacher feedback quality and teacher autonomy support, our finding concerning teacher feedback quantity was largely in line with a study by Trautwein and Lüdtke [20] where math homework expectancy was unrelated to teacher homework control. In their explanation, Trautwein and Lüdtke [20] (p. 255) posit that what may matter most to homework motivation (e.g., including homework expectancy) is the quality of teacher homework control, rather than the quantity of teacher homework control, adding that "researchers should bear this point in mind when constructing assessment instruments for future research". Thus, it is surprising and intriguing to find that math homework expectancy was unrelated to two teacher quality measures (feedback quality and autonomy support).

\subsection{Parent Variables}

Concerning parent variables, the fact that math homework expectancy was negatively associated with parent help quantity was congruent with the study by Trautwein and Lüdtke [20], where math homework expectancy was negatively associated with parental homework control. Likewise, the finding that math homework expectancy was unrelated to parent content support was in line with the study by Trautwein and Lüdtke [20], where math homework expectancy was unrelated to parental homework help. Furthermore, the present study extends the extant literature by showing that math homework expectancy was positively associated with parent autonomy support. Taken together, these results suggest that three parent variables (i.e., help quantity, content support, and autonomy support) have differential influences on math homework expectancy.

In light of the related finding that math homework expectancy was not associated with teacher autonomy support, the finding that math homework expectancy was positively associated with parent autonomy support implies that parent autonomy support may play a more important role in enhancing math expectancy. Given that no previous study, to our knowledge, has linked homework expectancy to both teacher autonomy support and parent autonomy support, there is a critical need to study the relative influence of two types of autonomy support (teacher vs. parent) on homework expectancy in future investigation.

\subsection{Other Motivation Variables}

The fact that math homework expectancy was positively related to math homework value is congruent with expectancy-value theory [1,11], Trautwein et al.'s homework model [6], and related empirical findings $[7,22]$. Additionally, the finding that math homework expectancy was positively associated with mastery-approach is congruent with the growing evidence concerning the positive influence of mastery-approach on self-efficacy $[16,48]$. Meanwhile, how do we explain the finding that math homework expectancy was negatively associated with performance-approach? One plausible explanation is that performance goals focusing on the demonstration of competence are associated with high anxiety and low self-efficacy [49]. This is likely to be more of a case for Chinese students, as Confucian-heritage culture places more emphasis on face-saving and public image $[50,51]$.

\subsection{Implications for Practice}

As this is the first study employing a multilevel approach to link the five clusters of variables to math homework expectancy, there is a need to continue a line of investigation such as this prior to providing more conclusive implications for practice. Having stated that, it appears that several important results in the current investigation might be helpful for practice.

First, results regarding homework variables (time, frequency, quality, interest, and favorability) suggest that it would be important to move beyond homework quantity to homework quality, interest, and favorability when designing and developing math homework assignments. Although this recommendation seems commonsensical, it is important to note that it is based on our findings after controlling for prior knowledge and confounding variables in other clusters, something that has been largely overlooked in prior empirical research. Hence, it would be important to design 
math homework assignments that adapt to students' learning needs and potentials (e.g., knowledge, skills, and competence) and interests (e.g., activity and content interests) [52]. This is especially important, as some students are more likely to struggle to follow through homework and online classes resulting from COVID-19 (e.g., digital inequalities) [21,53]. Furthermore, given our finding that math homework value was positively associated with math homework expectancy, it would be beneficial for teachers and parents to help students to better understand what math homework means to them (i.e., the instrumental value of doing math homework relating to their educational aspirations and future career goals). In addition, it would be important to encourage students to regularly plan and structure preferred activities during after-school hours. Consequently, they would be less bothered or torn by the temptation of wanting to engage in preferred activities during homework sessions, thus viewing homework assignments more favorably [25].

The finding that math homework expectancy was positively related to mastery-approach yet negatively to performance-approach suggests that it would be beneficial to place more emphasis on students' learning, improvement, and progress (as opposed to the demonstration of their competence) in math homework. However, this may present a challenge for middle school teachers, as previous studies indicate that it is more difficult to work with middle school teachers to develop a stronger mastery orientation than elementary school teachers [2].

Finally, our findings concerning parent variables (help quantity, content support, and autonomy support) imply that parents can still influence students' math homework expectancy at the middle school level; parent autonomy support, in particular (compared with help quantity and content support), plays a more beneficial role in promoting math homework expectancy. Hence, it would be important to encourage parents to provide children with autonomy support, by paying more close attention to their ideas and by expressing growing confidence in their capacities to complete math assignments by themselves. This type of autonomy support would be particularly important with girls, given our findingwas in line with the extant research $[19,20]$ that girls tend to have lower math homework expectancy.

\subsection{Strengths, Limitations, and Further Investigation}

Informed by Trautwein et al.'s homework model [6] and related literature [12,30,42,48,54], the current investigation employed multilevel models to link math homework expectancy to five clusters of variables (demographic, homework, teacher, parent, and motivation) involving a large sample of Chinese students. Our finding that homework expectancy was significantly related to at least one variable from four of five clusters provides empirical support to the homework model tested. This finding further illustrates the complexity of the factors influencing homework expectancy, something that has been largely overlooked in previous research on homework. Specifically, our findings point to the importance of incorporating (a) achievement goal theory and (b) other homework variables, including homework time, interest, and favorability (i.e., not just homework quality), in research on homework expectancy. Furthermore, our study raises an intriguing question concerning the relative influence of parents vs. teachers (e.g., autonomy support) on homework expectancy in further investigation.

Several limitations of the current investigation ought to be acknowledged. First, the word "effect" should be considered as "predictive effect" [6], and our investigation was correlational in nature. Additionally, our investigation is limited to math homework, as human motivation in general and with homework expectancy in particular is domain-specific [11,21]. Furthermore, even though great care was taken to include five clusters of variables, guided by Trautwein et al.'s model [21], achievement goal theory [18], and related literature [30], unobserved predictor variables (e.g., self-regulatory strategies and teachers' expectancies) might have influenced math homework expectancy had they been included.

Concerning further investigation, it would be important to examine the five clusters of variables included in the current investigation in different achievement domains (e.g., English, history, and physics). It would also be important to pursue this line of investigation in other countries (e.g., US, 
France, and Germany), as motivational beliefs (e.g., competence beliefs) can be shaped by cultural differences (e.g., norms, expectations, and socialization practices) [11,24,55]. Furthermore, there is a need to carry out longitudinal research to examine how these clusters of variables may influence homework expectancy over time (e.g., teacher autonomy support, parent autonomy support, and academic achievement). In addition, it would be informative to use experience sampling methodology to study how and under what conditions students' homework expectancy changes moment-to-moment while doing specific homework assignments. It would be particularly informative to investigate factors that influence homework expectancy in online environments, as (a) online homework presents both opportunities and challenges for students, parents, and teachers [37,54,56], and as (b) COVID-19 has forced "an unprecedented massive 'migration' from traditional in-class face-to-face education to online education" [57] (p. 113). Finally, given our findings concerning the differential predictive effects of (a) homework variables (time, frequency, quality, interest, and favorability), (b) goal orientations (masteryand performance-approaches), and (c) teacher variables vs. parent variables on homework expectancy, it is therefore highly desirable to undertake qualitative research to better understand the antecedents and consequences of homework expectancy from the perspectives of students, parents, and teachers.

Author Contributions: Conceptualization, J.X.; methodology, J.X.; software, W.C. and J.X.; formal analysis, C.W. and J.X.; investigation, C.W., J.D., and J.X.; data curation, J.D. and J.X.; resources, C.W.; writing-original-draft preparation, J.X.; writing-review and editing, C.W. and J.D.; supervision, J.D.; project administration, J.D. All authors have read and agreed to the published version of the manuscript.

Funding: Support for this research was provided in part by the University of Macau Start-Up Research Grant (SRG2019-00175-FED). The opinions expressed do not necessarily reflect the position or policy of the University of Macau, and no official endorsement should be inferred.

Conflicts of Interest: The authors declare no conflict of interest.

\section{References}

1. Eccles, J.S. Expectancies, values and academic behaviors. In Achievement and Achievement Motives: Psychological and Sociological Approaches; Spence, J.T., Ed.; Freeman: San Francisco, CA, USA, 1983; pp. 75-146.

2. Wigfield, A.; Eccles, J.S.; Fredricks, J.A.; Simpkins, S.; Roeser, R.W.; Schiefele, U. Development of achievement motivation and engagement. In Handbook of Child Psychology and Developmental Science, 7th ed.; Lamb, M.E., Lerner, R.M., Eds.; Wiley: Hoboken, NJ, USA, 2015; Volume 3, pp. 657-700.

3. Fan, H.; Xu, J.; Cai, Z.; He, J.; Fan, X. Homework and students' achievement in math and science: A 30-year meta-analysis, 1986-2015. Educ. Res. Rev. 2017, 20, 35-54. [CrossRef]

4. Fernández-Alonso, R.; Suárez-Álvarez, J.; Muñiz, J. Adolescents' homework performance in mathematics and science: Personal factors and teaching practices. J. Educ. Psychol. 2015, 107, 1075-1085. [CrossRef]

5. Rodríguez, S.; Núñez, J.C.; Valle, A.; Freire, C.; del Mar Ferradás, M.; Rodríguez-Llorente, C. Relationship between students' prior academic achievement and homework behavioral engagement: The mediating/moderating role of learning motivation. Front. Psychol. 2019, 10, 1047. [CrossRef] [PubMed]

6. Trautwein, U.; Lüdtke, O.; Schnyder, I.; Niggli, A. Predicting homework effort: Support for a domain-specific, multilevel homework model. J. Educ. Psychol. 2006, 98, 438-456. [CrossRef]

7. $\mathrm{Xu}, \mathrm{J}$. Homework Expectancy Value Scale for high school students: Measurement invariance and latent mean differences across gender and grade level. Learn. Individ. Differ. 2017, 60, 10-17. [CrossRef]

8. León, J.; Núñez, J.L.; Liew, J. Self-determination and STEM education: Effects of autonomy, motivation, and self-regulated learning on high school math achievement. Learn. Individ. Differ. 2015, 43, 156-163. [CrossRef]

9. Jacobs, J.E.; Lanza, S.; Osgood, D.W.; Eccles, J.S.; Wigfield, A. Changes in children's self-competence and values: Gender and domain differences across grades one through twelve. Child. Dev. 2002, 73, 509-527. [CrossRef]

10. Muenks, K.; Wigfield, A.; Eccles, J.S. I can do this! The development and calibration of children's expectations for success and competence beliefs. Dev. Rev. 2018, 48, 24-39. [CrossRef] 
11. Wigfield, A.; Eccles, J.S.; Schiefele, U.; Roeser, R.; Davis-Kean, P. Development of achievement motivation. In Handbook of Child Psychology, 6th ed.; Eisenberg, N., Ed.; Wiley: Hoboken, NJ, USA, 2006; Volume 3, pp. 933-1002.

12. Denissen, J.J.A.; Zarrett, N.R.; Eccles, J.S. I like to do it, I'm able, and I know I am: Longitudinal couplings between domain-specific achievement, self-concept, and interest. Child. Dev. 2007, 78, 430-447. [CrossRef]

13. Guo, J.; Marsh, H.W.; Parker, P.D.; Morin, A.J.; Yeung, A.S. Expectancy-value in mathematics, gender and socioeconomic background as predictors of achievement and aspirations: A multi-cohort study. Learn. Individ. Differ. 2015, 37, 161-168. [CrossRef]

14. Spinath, B.; Spinath, F.M.; Harlaar, N.; Plomin, R.P. Predicting school achievement from intelligence, self-perceived ability, and intrinsic value. Intelligence 2006, 34, 363-374. [CrossRef]

15. Wigfield, A.; Byrnes, J.B.; Eccles, J.S. Adolescent development. In Handbook of Educational Psychology, 2nd ed.; Alexander, P.A., Winne, P., Eds.; Erlbaum: Mahwah, NJ, USA, 2006; pp. 87-113.

16. Hulleman, C.S.; Senko, C. Up around the bend: Forecasts for achievement goal theory and research in 2020. In Advances in Motivation and Achievement; Urdan, T.C., Karabenick, S.A., Eds.; Emerald Group: Bingley, UK, 2010; Volume 16a, pp. 71-104.

17. Pintrich, P.R. Multiple goals, multiple pathways: The role of goal orientation in learning and achievement. J. Educ. Psychol. 2000, 92, 544-555. [CrossRef]

18. Conley, A.M. Patterns of motivation beliefs: Combining achievement goal and expectancy-value perspectives. J. Educ. Psychol. 2012, 104, 32-47. [CrossRef]

19. Dettmers, S.; Trautwein, U.; Lüdtke, O.; Kunter, M.; Baumert, J. Homework works if homework quality is high: Using multilevel modeling to predict the development of achievement in mathematics. J. Educ. Psychol. 2010, 102, 467-482. [CrossRef]

20. Trautwein, U.; Lüdtke, O. Predicting homework motivation and homework effort in six school subjects: The role of person and family characteristics, classroom factors, and school track. Learn. Instr. 2009, 19, $243-258$. [CrossRef]

21. Van Lancker, W.; Parolin, Z. COVID-19, school closures, and child poverty: A social crisis in the making. Lancet Public Health 2020, 5, 243-244. [CrossRef]

22. Yang, F.; Xu, J. Homework Expectancy Value Scale: Measurement invariance and latent mean differences across gender. J. Psychoeduc. Assess. 2018, 36, 863-868. [CrossRef]

23. Marsh, H.W.; Trautwein, U.; Lüdtke, O.; Köller, O.; Baumert, J. Academic selfconcept, interest, grades, and standardized test scores: Reciprocal effects models of causal ordering. Child. Dev. 2005, 76, 397-416. [CrossRef]

24. Xu, J. Reciprocal effects of homework self-concept, interest, effort, and math achievement. Contemp. Educ. Psychol. 2018, 55, 42-52. [CrossRef]

25. Xu, J. Models of secondary students' interest in homework: A multilevel analysis. Am. Educ. Res. J. 2008, 45, 1180-1205. [CrossRef]

26. $\mathrm{Xu}, \mathrm{J} . ; \mathrm{Wu}, \mathrm{H}$. Self-regulation of homework behavior: Homework management at the secondary school level. J. Educ. Res. 2013, 106, 1-13. [CrossRef]

27. Núñez, J.C.; Suárez, N.; Rosário, P.; Vallejo, G.; Cerezo, R.; Valle, A. Teachers' feedback on homework, homework-related behaviors, and academic achievement. J. Educ. Res. 2015, 108, 204-216. [CrossRef]

28. $\mathrm{Xu}, \mathrm{J}$. A study of the validity and reliability of the Teacher Homework Involvement Scale: A psychometric evaluation. Measurement 2016, 93, 102-107. [CrossRef]

29. Moroni, S.; Dumont, H.; Trautwein, U.; Niggli, A.; Baeriswyl, F. The Need to distinguish between quantity and quality in research on parental involvement: The example of parental help with homework. J. Educ. Res. 2015, 108, 417-431. [CrossRef]

30. Xu, J.; Fan, X.; Du, J.; He, M. A study of the validity and reliability of the Parental Homework Support Scale. Measurement 2017, 95, 93-98. [CrossRef]

31. Cooper, H.; Lindsay, J.J.; Nye, B.; Greathouse, S. Relationships among attitudes about homework, amount of homework assigned and completed, and student achievement. J. Educ. Psychol. 1998, 90, 70-83. [CrossRef]

32. Wigfield, A.; Cambria, J. Students' achievement values, goal orientations, and interest: Definitions, development, and relations to achievement outcomes. Dev. Rev. 2010, 30, 1-35. [CrossRef] 
33. Zimmerman, B.J.; Moylan, A.R. Self-regulation: Where metacognition and motivation intersect. In Handbook of Metacognition in Education; Hacker, D.J., Dunlosky, J., Graesser, A.C., Eds.; Routledge: New York, NY, USA, 2009; pp. 299-315.

34. Warton, P.M. The forgotten voices in homework: Views of students. Educ. Psychol. 2001, 36, 155-165. [CrossRef]

35. Xu, J.; Corno, L. Case studies of families doing third-grade homework. Teach. Coll. Rec. 1998, 100, 402-436.

36. Xu, J.; Yuan, R. Doing homework: Listening to students', parents', and teachers' voices in one urban middle school community. Sch. Community J. 2003, 13, 25-44.

37. $\mathrm{Xu}, \mathrm{J}$. Investigating factors that influence conventional distraction and tech-related distraction in math homework. Comput. Educ. 2015, 81, 304-314. [CrossRef]

38. Sun, M.; Du, J.; Xu, J.; Liu, F. Homework Goal Orientation Scale: Measurement invariance and latent mean differences across gender and grade level. Psychol. Sch. 2019, 56, 465-477. [CrossRef]

39. Raudenbush, S.; Bryk, A.S. Hierarchical Linear Models: Applications and Data Analysis, 2nd ed.; Sage: Thousand Oaks, CA, USA, 2002.

40. Carifio, L.; Perla, R. Resolving the 50 year debate around using and misusing Likert scales. Med. Educ. 2008, 42, 1150-1152. [CrossRef] [PubMed]

41. Norman, G. Likert scales, levels of measurement and the "laws" of statistics. Adv. Health Sci. Educ. 2010, 15, 625-632. [CrossRef]

42. Miller, A.D.; Murdock, T.B. Modeling latent true scores to determine the utility of aggregate student perceptions as classroom indicators in HLM: The case of classroom goal structures. Contemp. Educ. Psychol. 2007, 32, 83-104. [CrossRef]

43. Xu, J.; Yuan, R.; Xu, B.; Xu, M. Modeling students' interest in math homework. J. Educ. Res. 2016, 109, 148-158. [CrossRef]

44. Xu, J.; Du, J.; Wang, C.; Liu, F.; Huang, B.; Zhang, M.; Xie, J. Intrinsic motivation, favorability, time management, and achievement: A cross-lagged panel analysis. Unpublished work. 2020.

45. Ben-Eliyahu, A.; Linnenbrink-Garcia, L. Extending self-regulated learning to include self-regulated emotion strategies. Motiv. Emot. 2013, 37, 558-573. [CrossRef]

46. Ben-Eliyahu, A.; Bernacki, M.L. Addressing complexities in self-regulated learning: A focus on contextual factors, contingencies, and dynamic relations. Metacogn. Learn. 2015, 10, 1-13. [CrossRef]

47. Nadelson, L.; Hardy, K.K.; Yang, D. I like therefore I learn! Engineering student motivation to learn in their least and most favorite courses. In Proceedings of the ASEE Annual Conference \& Exposition, Seattle, WA, USA, 14-17 June 2015; 26, pp. 870.1-870.12.

48. Cellar, D.F.; Stuhlmacher, A.F.; Young, S.K.; Fisher, D.M.; Adair, C.K.; Haynes, S.; Riester, D. Trait goal orientation, self-regulation, and performance: A meta-analysis. J. Bus. Psychol. 2011, 26, 467-483. [CrossRef]

49. Senko, C.; Hulleman, C.S.; Harackiewicz, J.M. Achievement goal theory at the crossroads: Old controversies, current challenges, and new directions. Educ. Psychol. 2011, 46, 26-47. [CrossRef]

50. Du, J.; Xu, J.; Liu, F.; Huang, B.; Li, Z. Factors influence kindergarten teachers' emotion management in information technology: A multilevel analysis. Asia-Pac. Educ. Res. 2019, 28, 519-530. [CrossRef]

51. Hu, H.C. The Chinese concepts of "face". Am. Anthropol. 1944, 46, 45-64. [CrossRef]

52. Corno, L.; Xu, J. Doing homework as the job of childhood. Theory Pract. 2004, 43, 227-233. [CrossRef]

53. Beaunoyer, E.; Dupéré, S.; Guitton, M.J. COVID-19 and digital inequalities: Reciprocal impacts and mitigation strategies. Comput. Hum. Behav. 2020, 111, 106424. [CrossRef] [PubMed]

54. Xu, J.; Du, J.; Fan, X. "Finding our time": Predicting students' time management in online collaborative groupwork. Comput. Educ. 2013, 69, 139-147. [CrossRef]

55. Else-Quest, N.M.; Hyde, J.S.; Linn, M.C. Cross-national patterns of gender differences in mathematics: A meta-analysis. Psychol. Bull. 2010, 136, 103-127. [CrossRef] [PubMed] 
56. Magalhães, P.; Ferreira, D.; Cunha, J.; Rosário, P. Online vs traditional homework: A systematic review on the benefits to students' performance. Comput. Educ. 2020, 152, 103869. [CrossRef]

57. Bao, W. COVID-19 and online teaching in higher education: A case study of Peking University. Hum. Behav. Emerg. Technol. 2020, 2, 113-115. [CrossRef] 